\title{
Relaciones ciencia-tecnología-sociedad en la educación científica colombiana: una revisión del estado de la cuestión (2017-2021)
}

\author{
Science-Technology-Society Relationships in Colombian Science Education: A Review \\ of the State of the Art (2017-2021)
}

\section{Relações Ciência-Tecnologia-Sociedade na educação científica colombiana: uma revisão do estado da arte (2017-2021)}

\author{
Yisneth-Nathaniela Álvarez-Tobón ${ }^{1}$ \\ Dora-Inés Arroyave-Giraldo ${ }^{2}$ \\ Antonio García-Carmona ${ }^{3}$
}

Recibido: marzo de 2021

Aceptado: julio de 2021

Para citar este artículo: Álvarez-Tobón, Y. N., Arroyave-Giraldo, D. I., y García-Carmona, A (2021). Relaciones ciencia-tecnología-sociedad en la educación científica colombiana: una revisión del estado de la cuestión (20172021). Revista Científica, 42(3), 353-367. https://doi.org/10.14483/23448350.18231

\section{Resumen}

En este artículo se presentan los resultados de una investigación documental cuyo objetivo fue analizar las tendencias sobre el abordaje de las relaciones ciencia-tecnología-sociedad (CTS) en la educación científica en Colombia, entre los años 2017 y 2021. Se empleó, para ello, una metodología cualitativa basada en el análisis textual discursivo de los documentos seleccionados, con ayuda del software Nvivo11. Las categorías de análisis fueron: tipo de estudio, metodología, población, contexto didáctico CTS, e incorporación de las relaciones CTS en la educación científica. Entre otros resultados, se observó un empleo recurrente de la historia y la epistemología de la ciencia, así como el uso de controversias científicas, para abordar las relaciones CTS en la educación científica del contexto colombiano.

Palabras clave: ciencia-tecnología-sociedad; educación científica; investigación documental; tendencias.

\begin{abstract}
This article presents the results of a documentary research whose objective was to analyze the trends on the approach to science-technology-society (STS) relationships in science education in Colombia between 2017 and 2021. To this effect, a qualitative methodology was used, which was based on the discursive textual analysis of the selected documents
\end{abstract}

1. Universidad de San Buenaventura, Medellín, Colombia. Correo electrónico: yisneth.alvarez@ievillaflora.edu.co

2. Universidad de San Buenaventura, Medellín, Colombia. Correo electrónico: dora.arroyave@usbmed.edu.co

3. Universidad de Sevilla, España. Correo electrónico: garcia-carmona@us.es 
with the help of the Nvivo11 software. The analysis categories were: type of study, methodology, population, STS didactic context, and incorporation of STS relationships in science education. Among other results, a recurrent use of history and epistemology of science was observed, as well as the use of scientific controversies to address STS relationships in science education within the Colombian context.

Keywords: documentary research; science education; science-technology-society; trends.

\section{Resumo}

Este artigo apresenta os resultados de uma pesquisa documental cujo objetivo foi analisar as tendências da abordagem das relações Ciência-Tecnologia-Sociedade (CTS) na educação científica na Colômbia, entre os anos 2017-2021. Para isso, utilizou-se uma metodologia qualitativa baseada na análise textual discursiva dos documentos selecionados, com o auxílio do software Nvivo11. As categorias de análise foram: tipo de estudo, metodologia, população, contexto didático de DST e incorporação das relações CTS no ensino de ciências. Entre outros resultados, observou-se um uso recorrente da história e epistemologia da ciência, bem como o uso de controvérsias científicas, para abordar as relações CTS na educação científica no contexto colombiano.

Palavras-chaves: educação científica, ciência-tecnologia-sociedade, tendências, pesquisa documental.

\section{Introducción}

La comprensión de la relación ciencia-tecnología-sociedad (CTS) está considerada un pilar básico de la alfabetización científica deseable para la ciudadanía (Aikenhead, 2005a). La perspectiva CTS ubica a la ciencia en contextos sociales, tecnológicos, culturales, éticos y políticos, con el fin de que los estudiantes desarrollen concepciones más profundas del mundo en el que viven $y$, consecuentemente, lleguen a ser ciudadanos críticos y responsables (Bencze et al., 2020). Se trata, en definitiva, de una mirada educativa que busca humanizar la ciencia e incentivar la responsabilidad ciudadana (Vázquez-Alonso, Acevedo-Díaz y Manassero, 2005). En Colombia, puede decirse que la educación científica se muestra especialmente sensible a ello. Las relaciones CTS son referidas de manera explícita en el currículo oficial de ciencias naturales (Ministerio de Educación Nacional, 2006). Su atención educativa se promueve desde una perspectiva transversal, a lo largo de los distintos cursos y asignaturas de las etapas básica (ciencias naturales) y media (biología, química y física).

Las concepciones sobre CTS, en el contexto de la educación científica, han sido objeto de algunos estudios. En el ámbito iberoamericano, uno de los estudios con mayor alcance fue llevado a cabo en el marco de PIEARCTS (Proyecto Iberoamericano de Evaluación de Actitudes Relacionadas con la Ciencia, la Tecnología y la Sociedad) (Bennàssar et al., 2010), que evaluó las creencias y actitudes de muestras amplias de estudiantes y profesorado de diversos países de habla hispana y portuguesa. En el caso de Colombia, los resultados revelaron concepciones ingenuas sobre las relaciones CTS en profesores de ciencia en formación, en los primeros y últimos semestres.

Más recientemente, Osorio Marulanda (2019) ha analizado los principales fundamentos epistemológicos que subyacen en aquellos enfoques que la Organización de los Estados Iberoamericanos ha promovido respecto a la educación CTS. Los enfoques encontrados son: (i) controversias tecnocientíficas, (ii) participación pública de comunidades de ciencia y tecnología, y (iii) apropiación social de la ciencia por parte de la ciudadanía.

En Colombia un antecedente relevante es Quintero Cano (2010), quien analizó el proceso de gestión de la perspectiva CTS a escala internacional, y luego en este país. Dicho autor concluyó que es necesario involucrarla en las clases de ciencias mediante cuestiones sociocientíficas, reflexiones filosóficas, éticas, y sus relaciones con la educación ambiental.

Este contexto de antecedentes devela importantes estudios CTS realizados en el ámbito iberoamericano $y$, en menor medida, en Colombia, que 
hacen valiosos aportes a esta línea de trabajo en la didáctica de las ciencias. Sin embargo, Strieder, Bravo y Gil (2017) alertan de la importancia de revisar continuamente el tipo de tratamiento que se hace de CTS en las investigaciones educativas. No en vano, la educación CTS tiene una naturaleza evolutiva (Aikenhead, 2005a), que hace necesario determinar con regularidad cuáles son las orientaciones y objetivos más actuales al respecto.

Teniendo en cuenta esto, y dados los antecedentes bibliográficos sobre la educación CTS en el contexto de Colombia, se decidió llevar cabo una investigación documental orientada por la siguiente cuestión: ¿cuáles son las tendencias recientes (periodo 2017-2021) en la investigación relativa a la educación CTS en Colombia?

\section{Marco teórico}

El contexto ambiental y social en el que nos encontramos, en estos últimos años, exige replantear los objetivos de la educación en ciencias naturales. La pandemia por el virus Sars-CoV-2, por ejemplo, ha hecho evidente que nuestros estudiantes no están preparados para enfrentar la crisis sanitaria (Pietrocola et al., 2021), porque ante la cantidad de información que se generó desde los diferentes medios de comunicación, los estudiantes no recurren a la ciencia para validarla, sino a costumbres, creencias y opiniones sin argumentación. Es lo que Porlán (2018) Ilama "una mente dual", que consiste en una mente que actúa en las experiencias cotidianas, mientras que la mente académica se activa solo en los procesos escolares. Por ello, se insiste en la importancia de abordar la educación científica más allá de los contenidos disciplinares.

Buscando este objetivo, y a partir de los estudios sobre CTS, que surgen en la década de 1970 (Solbes, 2019), se empieza a prestar atención a ello en la educación científica. La larga trayectoria de tal propósito ha dado lugar a hablar de la tradición CTS para la enseñanza de las ciencias (CTS-EC) (Acevedo-Díaz y García-Carmona,
2016). Cabe mencionar también el uso del acrónimo CTSA debido a que algunos autores (p. ej., Pedretti y Nazir, 2011; Vilches et al., 2011) consideran relevante hacer explícita la ' $A$ ' del ambiente para que sea abordado con mayor intencionalidad. No obstante, es importante decir, al respecto, que la perspectiva ambiental ha sido tenida muy en cuenta en el enfoque CTS desde su origen; hasta el punto de que la atención al movimiento CTS en la educación científica estuvo originariamente inspirada por el ambientalismo y la sociología de la ciencia (Aikenhead, 2005a, p. 384). Por ello, otros autores entienden que añadir la ' $A$ ' como un apéndice al acrónimo no es realmente necesario (Acevedo-Díaz, Vázquez-Alonso y Paixâo, 2005) En este trabajo nos alineamos con esta última postura, y nos referiremos a 'CTS', tal y como ya hemos hecho en estudios anteriores (García-Carmona, 2008b, 2008a; Guerrero-Márquez y García-Carmona, 2020). Si bien, obviamente 'CTSA' será un término clave de búsqueda de documentos bibliográficos sobre la temática en este estudio.

Desde este marco teórico, se mencionan a continuación algunas consideraciones que se convirtieron en los dispositivos teóricos y metodológicos de la revisión documental Ilevada a cabo.

\section{CTS y naturaleza de la ciencia (NDC)}

La educación CTS más genuina se ha ocupado, desde sus inicios, de atender otro pilar esencial de la alfabetización científica: la comprensión de nociones básicas sobre la naturaleza de la ciencia (NDC) (Aikenhead y Ryan, 1992; Bencze et al., 2020). Esta responde a un conjunto de metaconocimientos sobre cómo se construye la ciencia, qué factores internos y externos a la comunidad científica influyen en ello, y cuáles son los rasgos más característicos del conocimiento científico. Desde la tradición CTS-EC, la NDC incluye aspectos epistémicos (esto es, aspectos racionales o cognitivos) y no-epistémicos (o sociológicos) de la ciencia (Acevedo-Díaz y García-Carmona, 2016). Una visión parecida de la NDC, en la que se integran 
aspectos de ambas dimensiones, se encuentra, por ejemplo, en los planteamientos de Allchin (2011) y Erduran y Dagher (2014); si bien, estos no se enmarcan explícitamente en la perspectiva CTS.

\section{CTS y cuestiones sociocientíficas (CSC)}

Las CSC se han ido incorporando al enfoque educativo CTS (Strieder, Bravo y Gil, 2017) y se consideran valiosas en la educación científica. Estas suelen tener una naturaleza controvertida; por tanto, su planteamiento en el aula implica el uso intencional de temas científicos que exigen que los estudiantes participen en discusiones y debates en los que deben examinar, sintetizar, argumentar y evaluar información; sopesar varios puntos de vista mientras analizan pruebas científicas y confrontan conflictos éticos (Karışan y Zeidler, 2017). Zeidler et al. (2005) consideran que las CSC van más allá de la educación CTS, entre otras razones, porque - según su parecer - esta última suele obviar aspectos morales, entre otros. Sin embargo, desde la tradición CTS-EC, inspirada en buena parte en los planteamientos de Aikenhead (2005a), los aspectos éticos y morales, que emergen en el marco de las complejas relaciones CTS, son también considerados. De modo que en este artículo se asume que las CSC están integradas dentro de los planteamientos del enfoque CTS.

\section{Incorporación de CTS en la educación científica}

Además de lo anterior, es importante conocer las formas en las que se incorpora la CTS en los programas curriculares. En este sentido, Aikenhead (2005a) se refiere a tres formas: (i) incorporación de contenidos CTS, de forma ocasional, en las clases de ciencias; (ii) enseñanza de la ciencia desde un enfoque CTS (o CTS como contexto para aprender contenidos de y sobre la ciencia), y (iii) enseñanza de contenidos CTS puros (principalmente en contextos de la filosofía y la sociología de la ciencia).

\section{Metodología}

Metodológicamente, el estudio planteado es de naturaleza interpretativa. Por ello, se acudió al método de análisis textual del discurso (ATD) (Moraes y Galiazzi, 2006), el cual oscila entre el análisis de contenido y el análisis del discurso, y cuya aplicación se apoya en el software cualitativo Nvivo11. Este método permitió establecer unas categorías de análisis, mediante un proceso dinámico y de depuración paulatina, a fin de responder al objetivo de la investigación: determinar las tendencias investigativas más recientes sobre educación CTS en Colombia. En consecuencia, el proceso de análisis tuvo dos momentos claves: el primero, correspondiente a la preparación de los documentos a partir de una serie de criterios de selección, que serán expuestos a continuación, y el segundo, al tratamiento investigativo de los documentos seleccionados a través del ATD.

Para llevar a cabo la selección de los documentos se llevaron a cabo los siguientes pasos:

(i) Artículos en revistas publicadas en el periodo 2017-2021:

- Selección de bases de datos de revistas: Scopus, ScienceDirect, Springer, Latindex, Web of Science, Scielo, Elsevier, ERIC, Google Académico, Redalyc, Dialnet y Colciencias.

- De esas bases de datos, se consultaron las revistas que siguen, las cuales fueron seleccionadas por su relevancia y gran difusión en el ámbito de la educación científica colombiana:

a. revistas nacionales: Científica, TED: Tecné, Episteme y Didaxis, Revista Colombiana de Ciencias Sociales, Bio-grafía, Praxis \& Saber, Pedagogía y Saberes, Educación y Ciencia, Educación y Educadores, Góndola, enseñanza y aprendizaje de las ciencias, Historia Crítica, Scientia et Technica.

b. revistas internacionales: Science \& Education, Revista Iberoamericana de Educación, 
Revista Eureka sobre Enseñanza y Divulgación de las Ciencias, Revista Iberoamericana de Ciencia, Tecnología y Sociedad, Journal of Chemical Information and Modeling, Enseñanza de las Ciencias, \#Tear: Revista de Educação, Ciência e Tecnologia, Educación Química, Tapuya: Latin American Science, Technology and Society, Investigações em Ensino de Ciencias.

- Establecimiento de palabras clave de búsqueda: ciencia-tecnología-sociedad, CTS o STS, CTSA o STSE, ciencia y tecnología, cuestiones sociocientíficas y CSC, educación en ciencias, educación científica y science education, enseñanza de las ciencias.

- Cribado para la inclusión de los artículos. Los artículos que serían incluidos en el análisis debían cumplir los siguientes requisitos: (i) el artículo está publicado en una revista colombiana, sin tener en cuenta si los autores son nacionales o internacionales; (ii) el artículo está publicado en una revista internacional, pero sus autores, filiación institucional y contexto de la investigación son colombianos; (iii) el artículo se encuentra disponible en acceso abierto para su consulta; y (iv) el artículo está publicado - como ya se ha avanzado - dentro del periodo 2017-2021.
La búsqueda inicial en las bases de datos indicadas dio como resultado un total de 824 artículos. Al aplicar los criterios de exclusión, se eliminaron 700 artículos, y otros 68 por duplicaciones, quedando finalmente una muestra de 56 artículos.

(ii) Tesis doctorales. En este caso, la búsqueda se hizo en los repositorios de las universidades colombianas, aplicando las mismas palabras clave utilizadas con los artículos y durante el periodo 2017-2021. La búsqueda se limitó igualmente al ámbito de la educación científica y se obtuvieron 15 investigaciones, de las cuales solo 10 se encontraban disponibles para su lectura completa.

Como resultado de esta búsqueda del proceso (i), resultó una muestra final de 66 documentos. Tras una lectura preliminar de sus resúmenes, fueron codificados con una "I" seguida del número de la investigación: 11, 12, 13... 166. Luego de la codificación, se procedió a la sistematización en el gestor bibliográfico Mendeley. Como último paso de este primer momento, los documentos organizados en el gestor se sistematizaron en el programa Nvivo11, cuyo resultado se recoge en la Tabla 1.

Una vez hecha la selección, se pasó al tratamiento de los documentos en Nvivo11 usando los procesos propios del ATD, que son: unitarización, categorización y metatextos. Mediante la

Tabla 1. Síntesis de las investigaciones seleccionadas

\begin{tabular}{|c|c|c|}
\hline Año & Código Investigaciones & Cantidad \\
\hline $\begin{array}{l}2021 \\
\text { enero-junio }\end{array}$ & (I6); (I26); (I30); (I41); (I52); (I53); (I54); (I59) & 8 \\
\hline 2020 & $\begin{array}{l}\text { (I1); (I4); (I7); (I10); (I17); (I31); (I34); (I35); (I36); (I37); } \\
\text { (I42); (I44); (I55); (I56); (I60) }\end{array}$ & 15 \\
\hline 2019 & (I3); (I9); (I16); (I19); (I22); (I28); (I29); (I32); (I57); (I58) & 10 \\
\hline 2018 & $\begin{array}{l}\text { (I2); (I11); (I13); (I21); (I24); (I27); (I39); (I40); (I43); (I45); } \\
\text { (I46); (I47); (I48); (I62); (I63); (I64); (I65) }\end{array}$ & 17 \\
\hline 2017 & $\begin{array}{l}\text { (I5); (I8); (I12); (I14); (I15); (I18); (I20); (I23); (I25); (I33); } \\
\text { (I38); (I49); (I50); (I51); (I61) }\end{array}$ & 15 \\
\hline
\end{tabular}

Fuente: elaboración propia 
unitarización se crearon las unidades de análisis, que permitieron la construcción de núcleos de significación, tales como: objetivos, tipo de estudio, marco teórico, metodología, población. Con la categorización, concebida como el conjunto de núcleos temáticos, se hizo un proceso de conjunción entre las categorías teóricas establecidas inicialmente (marco teórico) y las emergentes durante el proceso de categorización. Estas categorías se detallan en la Tabla 2. Como último proceso del ATD, se crearon los metatextos que, para el propósito de este artículo, se corresponden con las tendencias reflexionadas a partir del proceso de categorización, y que se explicitan en los resultados.

\section{Resultados}

A continuación, se abordan los principales resultados del análisis de cada uno de los 66 documentos seleccionados a partir de las cinco categorías y sus respectivas subcategorías. Producto de este tratamiento, se presentan también los aspectos o rasgos comunes que fueron creando los metatextos, es decir, las tendencias.

\section{Categoría 1. Tipo de estudios}

Se encontró que las investigaciones se centran en cinco objetivos base: revisión teórica $(18.1 \%)$, propuestas de intervención didáctica (33.3\%), propuestas de formación docente (21.2\%), metaanálisis (10.6\%) y concepciones y opiniones (16.6\%) (Figura 1).

Referente a las concepciones y opiniones acerca de CTS, se destaca el estudio I30: Marín (2021) en el que se evaluaron las concepciones sobre CTS en estudiantes de básica secundaria. Entre los metaanálisis, es de resaltar el estudio 146: Parga Lozano (2018) en el que realizó un análisis de los libros de química en Colombia, y se encontró que solo el 3\% de los documentos refieren la interacción CTS. A partir de la categoría se establecieron los siguientes metatextos (Tabla 3).

Tabla 2. Categorías de la investigación documental

\begin{tabular}{|c|c|c|}
\hline Categoría & Descripción & Subcategoría \\
\hline Tipo de estudio & $\begin{array}{l}\text { Centrados en: (i) caracterización, (ii) sobre las metaciencias (epistemología, } \\
\text { sociología, historia, psicología), (iii) sobre enseñanza-aprendizaje. }\end{array}$ & $\begin{array}{l}\text {-Concepciones/opiniones } \\
\text {-Metaciencias } \\
\text {-Enseñanza-aprendizaje }\end{array}$ \\
\hline Metodología & Focalizadas en: cualitativas, cuantitativas o mixtas. & $\begin{array}{l}\text {-Cualitativa } \\
\text {-Cuantitativa } \\
\text {-Mixta }\end{array}$ \\
\hline Población & $\begin{array}{l}\text { Contempla la población objeto de estudio, siendo los componentes de la } \\
\text { tríada profesores-estudiantes-contenido. }\end{array}$ & $\begin{array}{l}\text {-Estudiantes } \\
\text {-Estudiantes y profesores } \\
\text {-Profesores en formación } \\
\text {-Profesores en ejercicio } \\
\text {-Contenido }\end{array}$ \\
\hline $\begin{array}{l}\text { Contexto didáctico } \\
\text { CTS }\end{array}$ & $\begin{array}{l}\text { Dada la fundamental importancia de conocer el contexto didáctico bajo } \\
\text { el cual se hace la incorporación de las CTS en cada investigación, se } \\
\text { tuvieron en cuenta: episodios históricos, CTS para comprender NDC, } \\
\text { Cuestiones sociocientíficas, CTSA y CTS intercultural. }\end{array}$ & $\begin{array}{l}\text {-Episodios históricos } \\
\text {-CTS para comprender NDC } \\
\text {-CSC } \\
\text {-CTSA } \\
\text {-CTS intercultural }\end{array}$ \\
\hline $\begin{array}{l}\text { Incorporación } \\
\text { de CTS en la } \\
\text { educación } \\
\text { científica }\end{array}$ & $\begin{array}{l}\text { (i) Inserción CTS: su implementación es para motivar, o el desarrollo de } \\
\text { actividades puntuales. (ii) Contenidos disciplinares con enfoque CTS: "se } \\
\text { desarrollan cuando surgen los contenidos científicos" (Solbes, 2019, p. } \\
\text { 83). (iii) CTS puros: el contexto CTS se hace desde reflexiones filosóficas, } \\
\text { sociales, epistemológicas. }\end{array}$ & $\begin{array}{l}\text {-Inserción CTS } \\
\text {-Contenidos disciplinares con enfoque } \\
\text { CTS } \\
\text {-CTS puros }\end{array}$ \\
\hline
\end{tabular}

Fuente: elaboración propia 


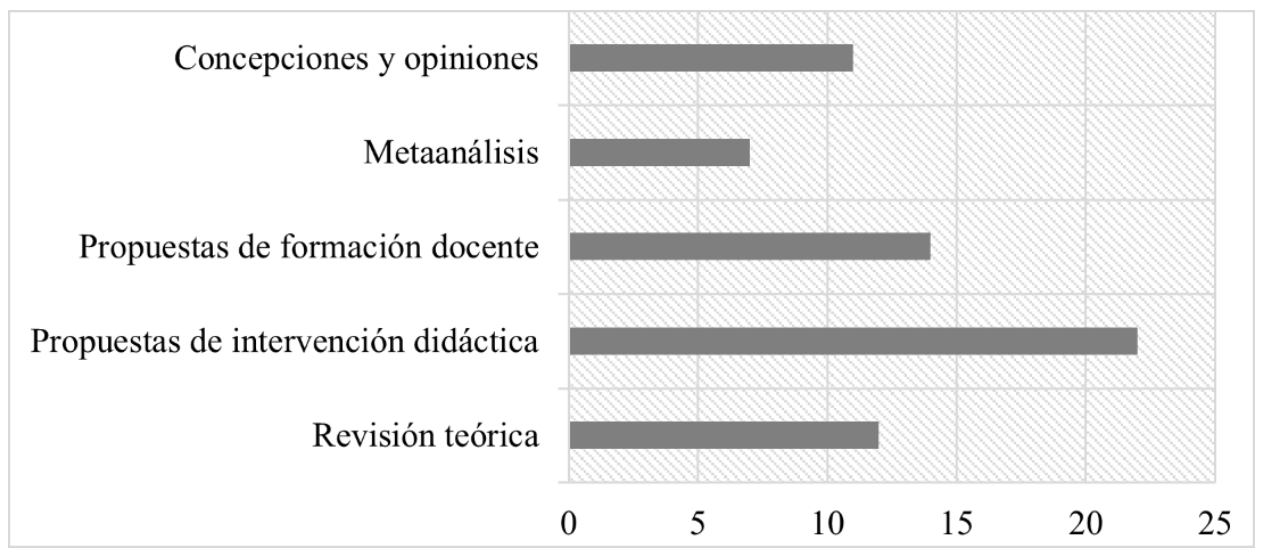

Figura 1. Tipo de estudio relacionados con la educación CTS en Colombia, periodo 2017-2021

Fuente: elaboración propia

Tabla 3. Tendencias en la categoría Tipo de estudio

\begin{tabular}{|c|c|c|}
\hline Subcategoría & Metatexto & Investigaciones \\
\hline \multirow{8}{*}{$\begin{array}{l}\text { Concepciones y } \\
\text { opiniones }\end{array}$} & \multirow{3}{*}{$\begin{array}{l}\text { Tipo de instrumento: uso de entrevistas y aplicación de } \\
\text { cuestionarios previamente diseñados o validación de nuevos } \\
\text { cuestionarios. }\end{array}$} & $\begin{array}{l}\text { COCTS (Cuestionario de Opiniones sobre } \\
\text { Ciencia, Tecnología y Sociedad): }(16.7 \%)\end{array}$ \\
\hline & & Cuestionarios tipo Likert: $(25 \%)$ \\
\hline & & Uso de entrevistas: $(58.3 \%)$ \\
\hline & \multirow{5}{*}{$\begin{array}{l}\text { Objeto CTS de la caracterización: existe una tendencia en } \\
\text { abordar definiciones. }\end{array}$} & Ciencia o tecnología: (33.3\%) \\
\hline & & Relación ciencia-género: (8.3\%) \\
\hline & & Interrelación CTSA: (33.3\%) \\
\hline & & Interrelación CTS: (8.3\%) \\
\hline & & Conceptos disciplinares: $(16.6 \%)$ \\
\hline \multirow{3}{*}{ Metaanálisis } & \multirow{3}{*}{$\begin{array}{l}\text { Foco CTS del metaanálisis: se evidencia interés de evaluar } \\
\text { publicaciones en revistas reconocidas. }\end{array}$} & Publicaciones (57.1\%) \\
\hline & & Textos didácticos (28.5\%) \\
\hline & & Grupos de investigación (14.2\%) \\
\hline \multirow{2}{*}{$\begin{array}{l}\text { Propuestas de } \\
\text { formación docente }\end{array}$} & \multirow{2}{*}{$\begin{array}{l}\text { Tipo de propuesta: (i) interdisciplinariedad, (ii) propuestas desde } \\
\text { estudios de caso. }\end{array}$} & Grupo de profesores: $(57.5 \%)$ \\
\hline & & Estudios de caso: $(42.4 \%)$ \\
\hline \multirow{2}{*}{$\begin{array}{l}\text { Propuestas de } \\
\text { intervención } \\
\text { didáctica }\end{array}$} & \multirow{2}{*}{$\begin{array}{l}\text { Es posible integrar este grupo de investigaciones en dos grandes } \\
\text { tendencias: (i) Instruccional vinculación CTS centrada en } \\
\text { competencias, aprendizaje de diferentes conceptos. (ii) No } \\
\text { instruccional centrada en los objetivos de alfabetización } \\
\text { científica, por ejemplo. }\end{array}$} & Instruccional (57.6\%) \\
\hline & & No instruccional (42.4\%) \\
\hline \multirow[b]{2}{*}{ Revisión teórica } & \multirow{2}{*}{$\begin{array}{l}\text { Se presentaron dos tendencias: } \\
\text { (i) Metaciencias (reflexión sobre asuntos epistemológicos, } \\
\text { históricos, sociológicos sobre CTS). (ii) Enseñanza CTS } \\
\text { (reflexiones sobre los contextos didácticos). }\end{array}$} & Metaciencias (90\%) \\
\hline & & Enseñanza CTS (10\%) \\
\hline
\end{tabular}

Nota: Se presenta la distribución de subcategorías desde las cuales se construyen las tendencias.

Fuente: elaboración propia 


\section{Categoría 2. Metodología}

El 94\% de las investigaciones se enmarcó en una metodología cualitativa, especialmente en estudios de caso y análisis documentales. Las dos investigaciones llevadas a cabo con metodología cuantitativa, 119: Ortega-Quevedo y Gil Puente (2019) e I40: Rodríguez y Suarez (2018), se centraron en diseños cuasiexperimentales mediante pretest y postest; solo dos trabajos se consideraron mixtos en tanto que usaron un cuestionario inicial y final con intervención didáctica intermedia, estas investigaciones mencionadas son 15: López Rivera (2017) e I51: Beltrán Martínez y Marín Quintero (2017).

\section{Categoría 3. Población}

En la Figura 2 se relaciona el tipo de población, que devela una mayoría en estudiantes. en especial aquellos pertenecientes a la básica secundaria. Otras investigaciones se centraron en los contenidos científicos escolares como objeto de su estudio, como es el caso de I3: Beltrán Castillo (2019), que analizó el racismo científico en los textos escolares de ciencias naturales en nuestro país. Por su parte, en el estudio 114: Medina Talero y Urazán Benítez (2017) se hizo una revisión del denominado "método científico" en la normatividad colombiana.

En el grupo de investigaciones de esta categoría se enmarca una importante tendencia con respecto al nivel educativo: universitaria $(28.7 \%)$ y preuniversitaria (68.1\%). Sin embargo, se halló el estudio I2: Rodríguez Hernández (2018), que involucró el trabajo de un grupo de investigación centrado en la interacción entre la universidad y la escuela. Otra tendencia en esta misma línea se refiere a: (i) educación formal (98\%), (ii) educación no formal $(1.5 \%)$. La única investigación relacionada como educación no formal es el caso de los clubes de ciencias en 111: Martínez Pérez, Vargas Sánchez y López Botello (2018).

\section{Categoría 4. Contexto didáctico CTS}

En esta categoría se tuvo en cuenta el contexto de enseñanza-aprendizaje en el que se hallaron en las investigaciones, siendo de cuatro tipos: episodios históricos, CSC, CTSA y CTS intercultural (Figura 3).

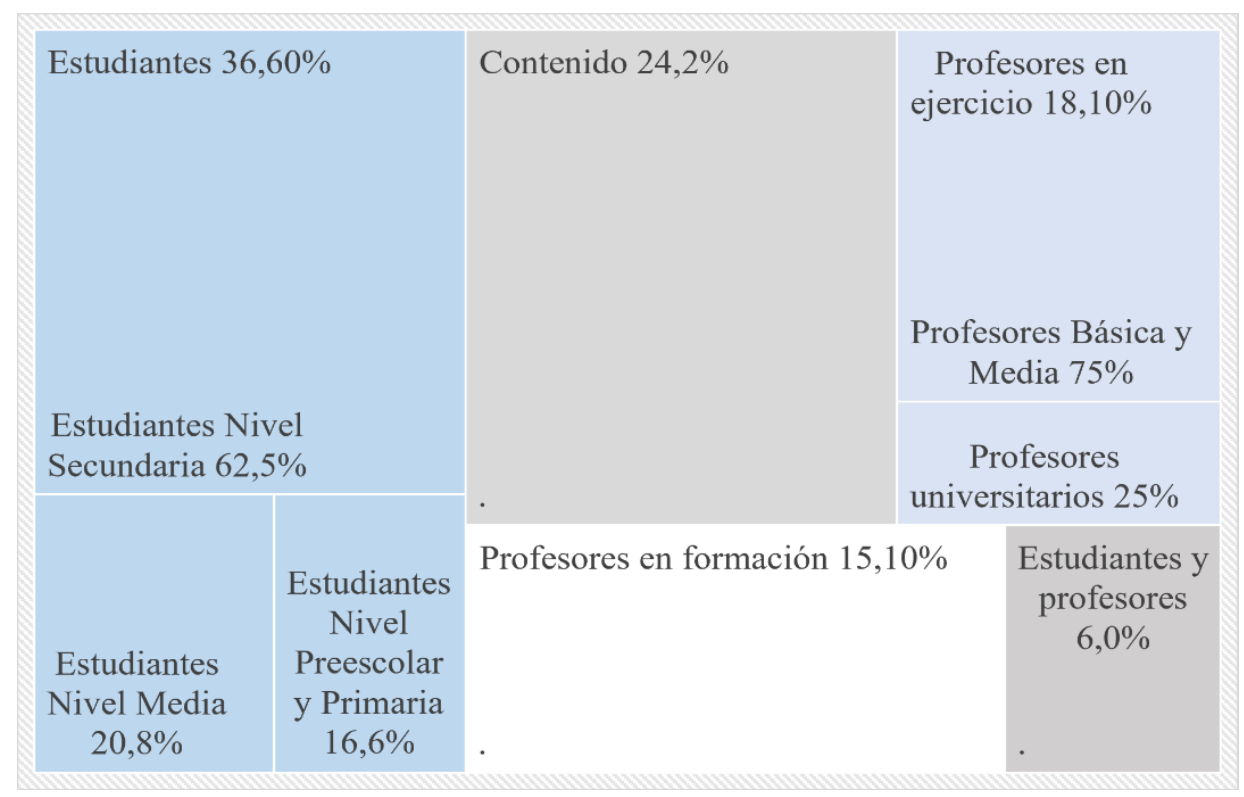

Figura 2. Tipo de población objeto de estudio en las investigaciones CTS

Fuente: elaboración propia 


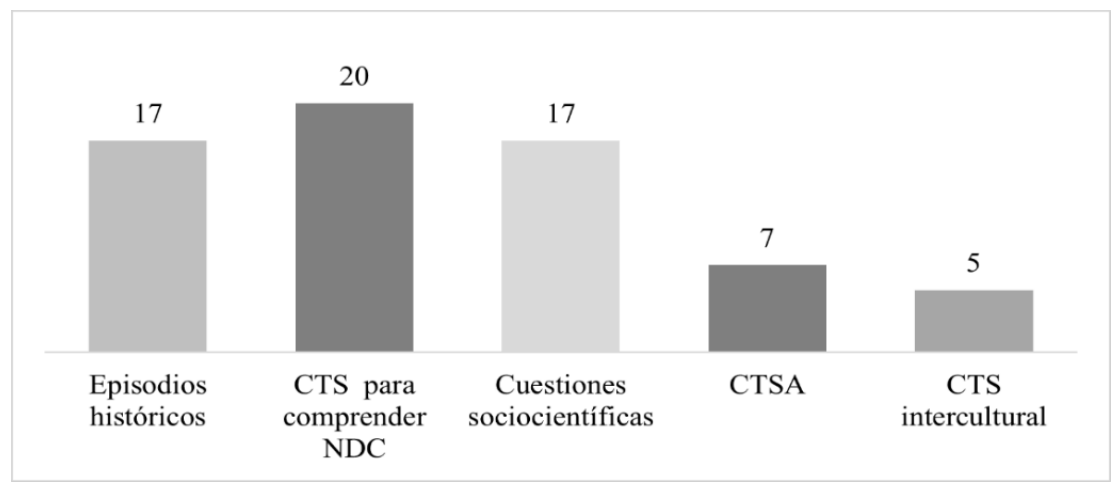

Figura 3. Contextos didácticos relacionados con la educación CTS

Fuente: elaboración propia

\section{Episodios históricos (25.7\%)}

Se evidencia la relevancia dada a la indagación histórica y epistemológica con fines pedagógicos, como es el caso de I38: Dueñas y Aristizábal-Fúquene (2017), que analizaron los tipos de tensiones y sus contribuciones, que se generan entre el conocimiento ancestral y el conocimiento científico respecto del caso histórico del oro prehispánico, de un grupo de estudiantes de décimo grado en la clase de química.

\section{CTS para comprender NDC (30.3\%)}

Estos estudios se enmarcan en el enfoque CTS para abordar aspectos sobre NDC como, por ejemplo, el estudio 126: Hansen et al. (2021) en el cual, desde un contexto CTS, se evaluaron la no neutralidad de la ciencia y la tecnología asumidos en las publicaciones de educación básica. Entre sus resultados se destacan visiones inadecuadas acerca de los desarrollos científicos y tecnológicos y del enfoque CTS.

\section{CSC en el marco educativo CTS (25.7\%)}

En esta subcategoría hay estudios relevantes como 164: Freire et al. (2018), en el que realizó un proceso de caracterización de cómo los profesores en formación representan el cambio climático como CSC a partir del diseño de secuencias didácticas.
Sus hallazgos señalan un manejo antropogénico del cambio climático.

\section{Perspectiva CTSA (10.6\%)}

Una investigación para destacar es 142: Alcocer y Hernández (2020), que recoge una exhaustiva revisión de la educación científica en Colombia a partir del análisis de 52 grupos de investigación colombianos y sus producciones hasta el año 2016. Respecto a CTS, hallaron que es una línea o temática presente en los eventos y las publicaciones; especialmente CTSA, por el interés pedagógico de articular la educación ambiental con las prácticas educativas.

\section{CTS intercultural $(7.5 \%)$}

Se hallaron estudios que se sustentaron en contextos de enseñanza alternativos muy interesantes como, por ejemplo, el trabajo 147: Bernal, Molina y Melo (2018), en el cual se crean puentes entre los conocimientos científicos escolares y los conocimientos tradicionales, respecto al caso de la papa con estudiantes de primaria.

\section{Categoría 5. Incorporación CTS en la educación científica}

Como se sustenta en el marco teórico, Aikenhead, 2005a plantea que, según la organización bajo 
la cual se dispone la incorporación de la CTS en los currículos, programas o estudios educativos, se puede determinar el grado o la profundidad en la relación entre las estructuras disciplinares y de CTS. En la Figura 4 se relaciona el tipo de incorporación en las investigaciones analizadas.

\section{Inserción CTS (9.1\%)}

La inserción CTS se caracteriza por complementar actividades con temáticas superficiales o puntuales sobre CTS. Un estudio representativo es I57: Carvalho Oliveira Marques y Cardoso Fernandes (2019), en el que los investigadores desarrollaron una secuencia didáctica para abordar el fenómeno de la luz con propósitos de su aplicación en la vida cotidiana. Sin embargo, tanto los cuestionarios que aplicaron antes y después como la intervención didáctica se centran en definiciones de la luz.

\section{Contenidos disciplinares con enfoque CTS (59.1\%)}

Este grupo de investigaciones se caracteriza por el desarrollo de contenidos de cualquiera de las disciplinas científicas y a partir de él se genera una estructura CTS desde, por ejemplo, problemáticas ambientales, cuestiones sociocientíficas, e interdisciplinariedad. Un caso ilustrativo de esta última es el trabajo 110: Mut et al. (2020). Sus autores toman como base los contenidos curriculares de un currículo de biología humana relacionados con la presión arterial para diseñar una secuencia didáctica desde una cuestión sociocientífica (mecanismos para su regulación).

\section{CTS puros $(31.8 \%)$}

En esta última subcategoría se abordan los fenómenos desde una revisión histórica y epistemológica de los conceptos que posibilita el tratamiento correlacional de las dimensiones epistemológica, sociológica y tecnológica. En esta línea, el documento I8: García Arteaga y Morcillo Molina (2017) ofrece una revisión histórica sobre la relación entre la experimentación y la microbiología. La reflexión se plantea a partir de los experimentos realizados por Louis Pasteur, y en ver cómo este episodio histórico se puede convertir en contexto para la enseñanza del papel de los microorganismos en la fermentación, abordando la relación CTS.

En estas dos últimas categorías (contexto didáctico CTS e incorporación CTS) se develaron dos

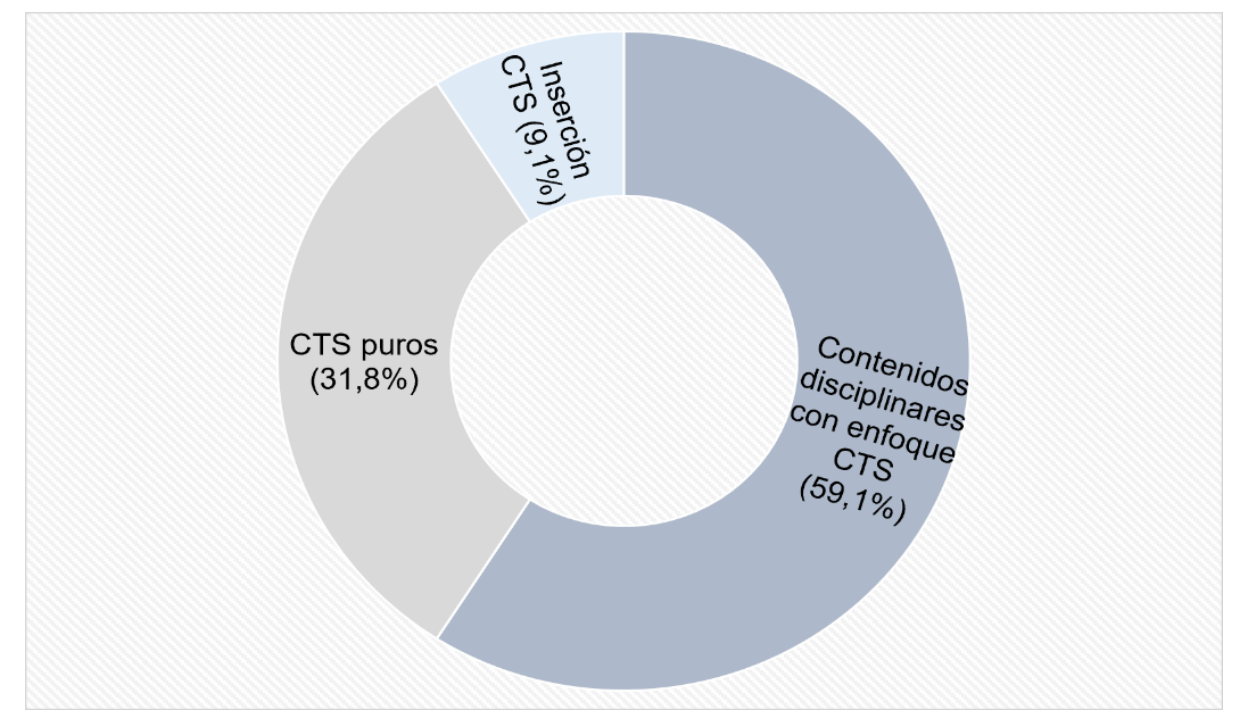

Figura 4. Incorporación CTS durante el periodo 2017-2021

Fuente: elaboración propia 
tendencias o metatextos, teniendo en cuenta los objetivos planteados en los estudios desde la educación CTS:

i. Estudios CTS con fin contextual, que ponen en contexto social los conocimientos y procesos científicos y tecnológicos que tengan utilidad en la cotidianidad (53.8\%). Muestra de ello es la investigación 121: Quintana, Páez y Téllez (2018), en la que se aplicó una propuesta denominada "actividades tecnológicas escolares" para explicar las características tanto técnicas como sociales en relación con las fuentes de energía renovables.

ii. Estudios CTS con fines críticos, orientados a la justicia social, o la sostenibilidad (46.2\%). En esta línea de propósitos sociales se destaca el documento 165: Rozo Dueñas y Martínez Pérez (2018), que, a partir de un trabajo de investigación-acción, ratifica las potencialidades de la formación investigadora con profesores de ciencias de educación preuniversitaria como acción sociopolítica al momento de tratar el cambio climático como CSC.

\section{Conclusiones}

La presente investigación documental, realizada a partir de los 66 trabajos seleccionados, brinda un panorama interesante para reflexionar sobre las tendencias en el abordaje de las relaciones CTS, dentro del contexto educativo colombiano. Los resultados permiten concluir que el enfoque CTS sigue evolucionando, y que existe un interés por parte de los investigadores de fomentar espacios académicos en la educación científica desde comprensiones cada vez más enriquecidas de las interacciones CTS.

Respecto a los tipos de estudio, se observa una tendencia en el desarrollo de propuestas de intervención didáctica y de formación docente, las cuales incluyen mayoritariamente en sus diseños metodológicos la caracterización inicial y final de concepciones y opiniones sobre CTS. Esto demuestra un gran interés propositivo que vuelve más robustos los objetivos del enfoque CTS en la educación científica. Las metodologías analizadas ponen también de manifiesto el alto número de estudios realizados en contextos preuniversitarios, lo cual surge de comprender la necesidad de poner en crisis la educación científica desde sus primeras etapas.

En lo referente al contexto didáctico para la educación CTS, se concluye una atención especial a episodios históricos. Es destacable que muchos de esos episodios son diseñados bajo la historia y cultura colombiana, tal y como sucede en el trabajo 125: Aristizábal Fúquene (2017), que muestra todo un programa de desarrollo docente bajo la reconstrucción sociohistórica del platino en Colombia desde el siglo XVIII, lo que demuestra una preocupación por abordar los aspectos sociológicos, tecnológicos y científicos de ese suceso. Este énfasis se muestra, además, en consonancia con los planteamientos de Bencze et al., 2020, cuando hablan de que "la corriente histórica proporciona análisis interesantes de cómo el material y los eventos históricos pueden usarse para enseñar las perspectivas de la naturaleza NDC (un componente importante de CTS)" (p. 831, traducción propia).

Se evidencia igualmente un creciente interés por las formas en las que interaccionan los conocimientos científicos y tradicionales del país, como las que ocurren en la comunidad wayuu, tema desarrollado en la investigación I4: Melo Brito (2020). Estudios como este reflejan que las visiones indígenas y alternativas de ver el mundo, son un proceso de enriquecimiento culturalmente poderoso, porque "están basados en el lugar, [son] monistas, holísticos, relacionales, misteriosos, dinámicos, sistemáticamente empíricos, basados en el tiempo cíclico, válidos, racionales y espirituales" (Gwekwerere, 2014, p.73, traducción propia).

En cuanto al tipo de incorporación CTS en la educación científica, se obtuvo que las investigaciones se concentran en los contenidos disciplinares con enfoque CTS, partiendo de contenidos curriculares que se articulan con temáticas 
relevantes CTS, tales como cuestiones sociocientíficas (CSC), problemas ambientales o las relacionadas con la NDC.

Respecto a los objetivos, se puede concluir que la mayoría de las investigaciones se concentra en los estudios CTS con propósitos de poner en un contexto social los conocimientos y procesos científico-tecnológicos que tengan utilidad en la cotidianidad.

Esta investigación documental representa, en la línea de las conclusiones descritas, un aporte a la didáctica de las ciencias, en la medida que evidencia el interés del contexto colombiano de avanzar en el conocimiento sobre la contextualización social de la ciencia y la tecnología. No obstante, cabe señalar una dificultad a la que se enfrentó la investigación. En los estudios revisados se presentan recurrentemente diferencias o ambigüedades con relación a los fundamentos teóricos, los propósitos y las conclusiones respecto a la educación CTS, que hicieron difícil su categorización. Pero ello representa, a su vez, una oportunidad para investigaciones futuras que realicen procesos de trazabilidad en las estructuras de las publicaciones.

Entre los retos que se establecen para la educación CTS en Colombia, se plantea la necesidad de seguir progresando hacia posturas o propósitos CTS más críticos y reflexivos como los relacionados con la justicia social (Hodson, 2021). También urge una evaluación respecto a los materiales educativos que utilizan los profesores para la educación en o desde CTS, puesto que estudios realizados con profesores en formación y en ejercicio indican un trato sesgado de conceptos de gran uso en algunas disciplinas, como por ejemplo el racismo científico, abordado en el estudio I3: Beltrán Castillo (2019). En esta línea, resulta interesante prestar atención a cómo las relaciones CTS se proyectan desde los medios de comunicación y en qué medida ello puede resultar un recurso útil para abordarlas en la educación científica (Guerrero-Márquez y García-Carmona, 2020). Finalmente, otro reto fundamental es avanzar hacia trabajos que promuevan la interacción universidad-escuela desde la creación de grupos de investigación, usando como marco o contexto de desarrollo la educación CTS.

\section{Referencias}

Acevedo-Díaz, J. A., García-Carmona, A. (2016). «Algo antiguo, algo nuevo, algo prestado». Tendencias sobre la naturaleza de la ciencia en la educación científica. Revista Eureka sobre Enseñanza y Divulgación de las Ciencias, 13(1), 3-19. https://doi.org/10.25267/Rev Eureka ensen divulg cienc.2016.v13.i1.02

Acevedo-Díaz, J. A., Vázquez-Alonso, Á., Paixâo, F. (2005). Educación CTS y alfabetización científica y tecnológica: una panorámica general a través de contextos culturales diferentes. CTS: Revista Iberoamericana de Ciencia, Tecnología y Sociedad, 2(6), 195-207

Aikenhead, G. (2005a). Educación Ciencia-Tecnología-Sociedad (CTS): una buena idea como quiera que se le Ilame. Educación Química, 16(2), 114-124. https://doi.org/10.22201/ fq.18708404e.2005.2.66121

Aikenhead, G. (2005b). Research into STS Science Education. Educación Química, 16(3), 384-397. https:// doi.org/10.22201/fq.18708404e.2005.3.66101

Aikenhead, G., Ryan, A. G. (1992). The development of a new instrument: "Views on science-technology-society" (VOSTS). Science Education, 76(5), 477-491

Alcocer Tocora, M., Hernández Hernández, C. (2020). Investigación en enseñanza de las ciencias en Colombia: estudio desde sus cosificaciones. Educación y Educadores, 23(1), 47-68. https://doi. org/10.5294/edu.2020.23.1.3

Allchin, D. (2011). Evaluating knowledge of the nature of (whole) science. Science Education, 95(3), 518542. https://doi.org/10.1002/sce.20432

Aristizábal Fúquene, C. A. (2017). Fortalecimiento de una identidad profesional docente mediante la interacción de una comunidad de desarrollo profesional a través del uso de la historia de la ciencia [Tesis de doctorado], Universidad Francisco José de 
Caldas, Bogotá D.C., Colombia. http://hdl.handle. net/11349/12933

Beltrán Castillo, M. J. (2019). Racismo científico en los textos escolares de ciencias naturales en Colombia [Tesis de doctorado], Universidad Francisco José de Caldas, Bogotá D.C., Colombia. http://hdl.handle. net/11349/15977

Beltrán Martínez, J., Marín Quintero, M. (2017). La historieta como material didáctico en la formación de actitudes relacionadas con la ciencia desde el abordaje de asuntos sociocientíficos. Enseñanza de las Ciencias, número extraordinario, 4715-4720

Bencze, L., Pouliot, C., Pedretti, E., Simonneaux, L., Simonneaux, J., Zeidler, D. (2020). SAQ, SSI and STSE education: Defending and extending "science-in-context". Cultural Studies of Science Education, 15(3), 825-851. https://doi.org/10.1007/ $\underline{\text { s11422-019-09962-7 }}$

Bennàssar, A., Vázquez, A., Manassero, M. A., García-Carmona, A. (Coor.). (2010). Ciencia, tecnología y sociedad en Iberoamérica: una evaluación de la comprensión de la naturaleza de ciencia y tecnología. OEI

Bernal, M. C., Molina Andrade, A., Melo, N. B. (2018). 6B020 Puente contextual, dialogo de conocimientos tradicionales y científicos escolares: el caso de la papa en el grado cuarto de primaria. Tecné, Episteme y Didaxis: TED, extraoridinario, 1-9

Carvalho Oliveira Marques, C. V., Cardoso Fernandes, D. (2019). Luz e cotidiano: ideias prévias de alunos do ensino fundamental sob a perspectiva da alfabetização científica. Góndola, enseñanza y aprendizaje de las ciencias, 14(2), 268-285. https://doi. org/10.14483/23464712.13704

Dueñas, Y., Aristizábal-Fúquene, A. (2017). Saber ancestral y conocimiento científico: tensiones e identidades para el caso del oro en Colombia. Tecné, Episteme y Didaxis: TED, 42, 25-42. https://doi. org/10.17227/01203916.6961

Erduran, S., Dagher, Z. R. (2014). Reconceptualizing the Nature of Science for Science Education: Scientific Knowledge, Practices and Other Family Categories (Contemporary Trends and Issues in Science
Education, vol. 43). Springer Netherlands. https:// doi.org/10.1007/978-94-017-9057-4

Freire, L. M., Brück, M., Juliani, S. de F., Benac, R. M. (2018). S202 Investigamos como professores em formação inicial representam controvérsias sociocientíficas (CS). Uma oficina sobre o tema mudanças climáticas (MC) foi cenário empírico. A atividade final foi a elaboração de uma sequência didática para desenvolver o. Tecné, Episteme y Didaxis: TED, (Extraordin), 1-6

García-Arteaga, E. G., Morcillo Molina, C. (2017). Actividad histórico-experimental en microbiología para la enseñanza de las ciencias. En Á. E. Romero-Chacón (Ed.), La experimentación en la clase de ciencias (pp. 79-98). Universidad de Antioquia

García-Carmona, A. (2008a). Relaciones CTS en la educación científica básica. I. Un análisis desde los textos escolares en la enseñanza de la electrónica. Enseñanza de las Ciencias, 26(3), 375-388. https:// doi.org/10.5565/rev/ensciencias.3749

García-Carmona, A. (2008b). Relaciones CTS en la educación científica básica. II. Investigando los problemas del mundo. Enseñanza de las Ciencias, 26(3), 389-402

Guerrero-Márquez, I.. García-Carmona, A. (2020). La energía y su impacto socioambiental en la prensa digital: temáticas y potencialidades didácticas para una educación CTS. Revista Eureka sobre Enseñanza y Divulgación de las Ciencias, 17(3), 617-627. https://doi.org/10.25267/Rev_Eureka_ensen_divulg_cienc.2020.v17.i3.3301

Gwekwerere, Y. (2014). Bridging Culture, Indigenous and Scientific Ways of Knowing. Cultural and Pedagogical Inquiry, 6(2). https://doi.org/10.18733/ C3GW24

Hansen, T. R., Marsango, D., Dos Santos, R. A. (2021). A presença da não neutralidade da ciência-tecnologia em literatura sobre a educação básica. Góndola, enseñanza y aprendizaje de las ciencias, 16(2), 238-254. https://doi. org/10.14483/23464712.15823

Hodson, D. (2021). Going beyond STS education: Building a curriculum for sociopolitical activism. Canadian Journal of Science, Mathematics and 
Technology Education, 20(4), 592-622. https://doi. org/10.1007/s42330-020-00114-6

Karışan, D., Zeidler, D. L. (2017). Contextualization of nature of science within the socioscientific issues framework: A review of research. International Journal of Education in Mathematics, Science and Technology, 5(2), 139-152.

López Rivera, Z. C. (2017). Innovación didáctica para la apropiación de la ciencia y la tecnología en estudiantes de educación básica y media [Tesis de doctorado], Universidad de La Salle, Bogotá D.C., Colombia. https://ciencia.lasalle.edu.co/ doct_educacion_sociedad/14/

Marín, G. (2021). Algunas concepciones CTSA de estudiantes de noveno grado sobre conceptos termodinámicos. Tecné, Episteme y Didaxis: TED, 49, 239-254. https://doi.org/10.17227/ted.num49-8645

Martínez Pérez, L. F., Vargas Sánchez, D. L., López Botello, J. A. (2018). Los clubes de ciencia: un análisis discursivo sobre la negociación de significados científicos y tecnológicos en un centro interactivo. Tecné, Episteme y Didaxis: TED, 43, 67-90. https:// doi.org/10.17227/ted.num43-8652

Medina Talero, N. A., Urazán Benítez, R. (2017). La naturaleza de las ciencias y la metodología de trabajo científico: una lectura a través de la normatividad colombiana. Bio-grafía, extraordinario: IV Congreso Nacional de Investigación en Enseñanza de la Biología, 134-145. https://doi.org/10.17227/bio-grafia. extra2017-7091

Melo Brito, N. B. (2020). Puentes entre conocimientos científicos escolares y conocimientos ecológicos tradicionales: Un estudio de aula en la comunidad wayuu [Tesis doctoral], Universidad Distrital Francisco José de Caldas, Bogotá D.C., Colombia. http://hdl.handle.net/11349/24851

Ministerio de Educación Nacional. (2006). Derechos básicos de aprendizaje. Ciencias Naturales. Ministerio de Educación Nacional

Moraes, R., Galiazzi, M. do C. (2006). Análise textual discursiva: processo reconstrutivo de múltiplas faces. Ciência \& Educação, 12(1), 117-128

Mut, P. N., Bahamonde, N., Lozano, E. E., Cremer, M. C. (2020). El desarrollo de una línea disciplinar para la enseñanza del modelo de presión arterial en la formación del profesorado en biología. Tecné, Episteme y Didaxis: TED, 47, 143-159. https://doi. org/10.17227/ted.num47-7920

Ortega-Quevedo, V., Gil Puente, C. (2019). La naturaleza de la ciencia y la tecnología: una experiencia para desarrollar el pensamiento crítico. Científica, 35(2), 167-182. https://doi. org/10.14483/23448350.14095

Osorio Marulanda, C. (2019). La educación CTS: un espacio para la cooperación iberoamericana. Revista Iberoamericana de Ciencia, Tecnología y Sociedad - CTS, 14(42), 99-114

Parga Lozano, D. L. (2018). Investigaciones en Colombia sobre libros de texto de química: análisis documental. Tecné, Episteme y Didaxis: TED, 44, 111-128. https://doi.org/10.17227/ted. num44-8992

Pedretti, E., Nazir, J. (2011). Currents in STSE education: Mapping a complex field, 40 years on. Science Education, 95(4), 601-626. https://doi. org/10.1002/sce.20435

Pietrocola, M., Rodrigues, E., Bercot, F., Schnorr, S. (2021). Risk society and science education. Science \& Education, 30(2), 209-233. https://doi. org/10.1007/s11191-020-00176-w

Porlán, R. (2018). Didáctica de las ciencias con conciencia. Enseñanza de las Ciencias, 36(3), 5-22. https://doi.org/10.5565/rev/ensciencias.2795

Quintana, A., Páez, J. J., Téllez, P. (2018). Actividades tecnológicas escolares: un recurso didáctico para promover una cultura de las energías renovables. Pedagogía y Saberes, 48, 43-57. https://doi. org/10.17227/pys.num48-7372

Quintero Cano, C. A. (2010). Enfoque Ciencia, Tecnología y Sociedad (CTS): perspectivas educativas para Colombia. Zona Próxima, 12, 222-239.

Rodríguez Hernández, B. F. (2018). Conocimiento profesional del profesor de ciencias al abordar cuestiones sociocientíficas: un estudio de caso de un grupo de investigación en la interacción universidad-escuela [Tesis de doctorado], Universidad Pedagógica Nacional, Bogotá D.C., Colombia. http:// hdl.handle.net/20.500.12209/9303 
Rodríguez, I. B., Suarez, O. J. (2018). 2B044 Argumentación al tratar un caso sociocientífico en una secuencia didáctica que incorpora PodCast. En: VIII Congreso Internacional sobre Formación de Profesores de Ciencias

Rozo Dueñas, O., Martínez Pérez, L. (2018). S304 Formación del profesor como investigador desde la escuela: fundamental acción sociopolítica y ambiental a partir del abordaje del cambio climático. En: VIII Congreso Internacional sobre Formación de Profesores de Ciencias

Solbes, J. (2019). Cuestiones socio-científicas y pensamiento crítico: una propuesta para cuestionar las pseudociencias. Tecné, Episteme y Didaxis: TED， 46, 81-99. https://doi.org/10.17227/ted. num46-10541

Strieder, R. B., Bravo-Torija, B., Gil Quílez, M. J. (2017). Ciencia-tecnología-sociedad: ¿qué estamos haciendo en el ámbito de la investigación en educación en ciencias? Enseñanza de las Ciencias, 35(3), 29-49. https://doi.org/10.5565/rev/ ensciencias. 2232

Vázquez-Alonso, Á., Acevedo-Díaz, J. A., Manassero Mas, M. A. (2005). Más allá de la enseñanza de las ciencias para científicos: hacia una educación científica humanística. Revista Electrónica de Enseñanza de las Ciencias, 4(2), 1-30

Vilches, A., Gil Pérez, D., Praia, J. (2011). De CTS a CTSA: educación por un futuro sostenible. En W. L. Pereira y D. Auler (Eds.), CTS e educação científica: desafios, tendências e resultados de pesquisa (pp. 185-209). Universidade de Brasília

Zeidler, D. L., Sadler, T. D., Simmons, M. L., Howes, E. V. (2005). Beyond STS: A research-based framework for socioscientific issues education. Science Education, 89(3), 357-377. https://doi.org/10.1002/sce.20048 